\title{
Thermodynamic Properties of Atactic Polypropylene Solutions
}

\author{
Hiroshi OCHIAI, ${ }^{*}$ Toshihiko OHASHI, ${ }^{*}$ Yuji TADOKORO,** \\ and Ichiro MURAKAMI* \\ * Department of Chemistry, Faculty of Science, Hiroshima University, \\ Higashisenda-machi, Naka-ku, Hiroshima 730, Japan. \\ **Chemistry Laboratory, Faculty of Integrated Arts and Sciences, \\ Hiroshima University, Higashisenda-machi, \\ Naka-ku, Hiroshima 730, Japan.
}

(Received November 20, 1981)

\begin{abstract}
The heats of mixing of atactic polypropylene at infinite dilution were measured at $25^{\circ} \mathrm{C}$ in linear alkanes, aromatic hydrocarbons, cyclohexane, chloroform, and carbon tetrachloride. For some solvent systems excess volume and chemical potential measurements were also performed at $25^{\circ} \mathrm{C}$. The results were treated by the new Flory theory for polymer solutions. The exchange interaction parameter $X_{12}$ increases with an increase in the chain length of $n$-alkanes and decreases with the introduction of aliphatic groups into benzene. These changes can be interpreted well in terms of the difference in force field between methyl-methylene contacts or aliphatic-aromatic contacts. The observed excess volumes are negative for $n$-alkanes and positive for rather globular cyclohexane, chloroform, carbon tetrachloride, and benzene, which is consistent with the prediction by the theory. However, the calculated excess volumes are less than those observed for all systems studied. Agreement between experimental and theoretical $\chi$-parameters can be achieved by the introduction of the exchange entropy parameter $Q_{12}$ and adjusting the contact surface ratio $\left(s_{2} / s_{1}\right)$.

KEY WORDS Thermodynamics / Polypropylene / Exchange Interaction Parameter / Heats of Mixing / Excess Volume / $\chi$-Parameter /
\end{abstract}

The concentration dependence of the $\chi$ parameter, lower critical solution temperature (LCST), volume change and exothermic heat of mixing of polymer solutions were beyond the scope of conventional polymer solution theories. Thermodynamic theories based on the free volume concept have enabled us to explain these interesting and unsolved problems. Patterson et al. ${ }^{1,2}$ succeeded in explaining the LCST phenomenon by applying the cell model to polymer solutions. Flory et al. ${ }^{3,4}$ also proposed a new theory applicable to various non-polar polymer solutions, and explained the concentration dependence of the $\chi$-parameter and volume change on mixing. Both theories emphasize the importance of the effects of the pure component properties on the solution properties, e.g., the differences in "free volume" and "force field" between polymer and solvent. The former difference gives an exothermic effect to the heat of mixing and the latter gives an endothermic effect.
Atactic polypropylene (APP) is a non-polar amorphous polymer and is of interest from the stand point of polymer solution property analysis, similar to polyisobutylene (PIB). However, the thermodynamic properties of APP solution have so far recieved little attention. ${ }^{5,6}$ In the present study, the heats of mixing, excess volume and vapor pressure measurements were carried out at $25^{\circ} \mathrm{C}$ for the APP-various solvent systems, and the results are discussed on the basis of the new Flory theory.

\section{EXPERIMENTAL}

\section{Materials}

Atactic polypropylene (APP) was obtained as an ether soluble material of conventional polypropylene and provided by Sumitomo Chemicals Industries Ltd. The polymer was made into three crude fractions using benzene-methanol solutions. The middle fraction was repeatedly purified by 
precipitation and recovered by freeze-drying from a benzene solution. The molecular weight estimated from intrinsic viscosity was about $1.8 \times 10^{4}$.

The solvents used here were six linear alkanes $(n$ $\mathrm{C}_{5}-n-\mathrm{C}_{10}$ ), six aromatic hydrocarbons (benzene, toluene, ethylbenzene, and $o-, m-, p$-xylenes), cyclohexane, chloroform, and carbon tetrachloride. All the solvents were purified by ordinary methods prior to use.

\section{Measurements}

Solvent activity was measured by vapor sorption using apparatus similar to that of Tait. ${ }^{7}$ Accuracy was within $\pm 0.1 \mathrm{mmHg}$ for vapor pressure and $\pm 0.5 \mathrm{mg}$ for solvent uptake.

Heats of mixing at infinite dilution were determined by a twin-type conduction microcalorimeter (Tokyo-Riko), which is well suited for the measurement of slow thermal effects such as polymer dissolution. Five to ten runs were made, and the experimental values of $h_{\infty}^{\mathrm{E}}$ expressed in $\mathrm{Jg}^{-1}$ were averaged. Average deviations were $\pm 5 \%$ for the largest heats and $\pm 10 \%$ for the smallest heats.

Excess volume was estimated from the density values of mixtures measured with a glass pycnometer, consisting of a bulb of sufficient volume (about $10 \mathrm{ml}$ ) and a calibrated capillary $(20 \mathrm{~cm}$ long and $0.8 \mathrm{~mm}$ in diameter). Accuracy was about $2 \times 10^{-4} \mathrm{~g} \mathrm{ml}^{-1}$.

\section{Theory}

According to the new Flory theory, the equationof-state for a pure liquid and mixture, expressed in its reduced form, is given by,

$$
\tilde{P} \tilde{v} / \tilde{T}=\tilde{v}^{1 / 3} /\left(\tilde{v}^{1 / 3}-1\right)-1 / \tilde{v} \tilde{T}
$$

The heat of mixing per gram of polymer at infinite dilution is,

$$
\begin{aligned}
h_{\infty}^{\mathrm{E}}=P_{2}^{*} v_{2}^{*} & {\left[\left(\tilde{v}_{2}^{-1}-\tilde{v}_{1}^{-1}\right)-\alpha_{1} T / \tilde{v}_{1}\left(1-T_{1}^{*} / T_{2}^{*}\right)\right] } \\
& +\left(s_{2} / s_{1}\right)\left(v_{2}^{*} / \tilde{v}_{1}\right) X_{12}\left(1+\alpha_{1} T\right)
\end{aligned}
$$

The $\chi$-parameter and excess volume of mixing are given as follows:

$$
\begin{aligned}
\left(\mu_{1}-\mu_{1}^{0}\right)^{\mathrm{R}}= & R T \varphi_{2}^{2} \chi \\
= & P_{1}^{*} V_{1}^{*}\left\{3 \tilde{T}_{1} \ln \left[\left(\tilde{v}_{1}^{1 / 3}-1\right) /\left(\tilde{v}^{1 / 3}-1\right)\right]\right. \\
& \left.+\left(\tilde{v}_{1}^{-1}+\tilde{v}^{-1}\right)\right\}+V_{1}^{*} X_{12} \theta_{2}^{2} / \tilde{v} \\
& \tilde{v}^{\mathrm{E}}=\tilde{v}-\tilde{v}^{0}, \quad \tilde{v}^{\mathrm{E}} / \tilde{v}^{0}=\Delta v / v^{0}
\end{aligned}
$$

where

$$
\tilde{v}^{0}=\varphi_{1} \tilde{v}_{1}+\varphi_{2} \tilde{v}_{2}
$$

The reduced volume $\tilde{v}$ at a given composition is obtained as the solution of eq 1 for $p=0$, using the reduced temperature $\tilde{T}$ for the mixture:

$$
\widetilde{T}=\left(\varphi_{1} P_{1}^{*} \widetilde{T}_{1}+\varphi_{2} P_{2}^{*} \widetilde{T}_{2}\right) /\left(\varphi_{1} P_{1}^{*}+\varphi_{2} P_{2}^{*}-\varphi_{1} \theta_{2} X_{12}\right)
$$

In these equations, the subscripts 1 and 2 express the solvent and polymer components, respectively. The tiled variables $\tilde{v}, \tilde{P}$, and $\tilde{T}$ are defined as $\tilde{v}=$ $v / v^{*}, \tilde{P}=P / P^{*}$, and $\tilde{T}=T / T^{*}$ where $v^{*}, P^{*}$, and $T^{*}$ are the respective reduction parameters. The symbol $s$ is the number of contact sites per segment (proportional to the surface area per segment), $\alpha$ is the thermal expansion coefficient, $X_{12}$ is the exchange interaction parameter related to the difference in force field between solvent and polymer. $\varphi$ and $\theta$ are the segment fraction and the surface fraction, respectively.

The reduction parameters $v^{*}, P^{*}$, and $T^{*}$ for

\begin{tabular}{|c|c|c|c|c|}
\hline \multirow{2}{*}{ Component } & $v^{*}$ & $T^{*}$ & $P^{*}$ & $S$ \\
\hline & $\mathrm{cm}^{3} \mathrm{~g}^{-1}$ & $\mathrm{~K}$ & $\mathrm{~J} \mathrm{~cm}^{-3}$ & $A^{-1}$ \\
\hline Polypropylene & 0.9661 & 5930 & 468.8 & 0.64 \\
\hline$n$-Pentane & 1.1829 & 4158 & 406.4 & 1.08 \\
\hline$n$-Hexane & 1.1559 & 4445 & 423.6 & 1.04 \\
\hline$n$-Heptane & 1.1346 & 4654 & 429.0 & 1.01 \\
\hline$n$-Octane & 1.1180 & 4826 & 433.2 & 0.99 \\
\hline$n$-Nonane & 1.1072 & 4993 & 435.3 & 0.98 \\
\hline$n$-Decane & 1.0944 & 5095 & 445.8 & 0.95 \\
\hline Cyclohexane & 1.0012 & 4720 & 531.6 & 0.93 \\
\hline Benzene & 0.8860 & 4709 & 627.8 & 0.99 \\
\hline Toluene & 0.9784 & 5025 & 560.9 & 0.91 \\
\hline Ethylbenzene & 0.9806 & 5202 & 540.0 & 0.87 \\
\hline$o$-Xylene & 0.9731 & 5342 & 540.0 & 0.87 \\
\hline$m$-Xylene & 0.9872 & 5267 & 540.0 & 0.87 \\
\hline$p$-Xylene & 0.9872 & 5194 & 531.6 & 0.87 \\
\hline $\mathrm{CHCl}_{3}$ & 0.5197 & 4625 & 565.0 & 0.97 \\
\hline $\mathrm{CCl}_{4}$ & 0.4890 & 4720 & 564.0 & 1.03 \\
\hline
\end{tabular}
APP and solvents were evaluated from the density, thermal expansion coefficient and thermal pressure coefficient in their pure state (Table I). The necessary data were obtained from ref 5 and 8 for APP, and in ref 9,10 , and 11 for solvents. The values of $s$ was estimated from the ratio of surface area-to-

Table I. Equation-of-state parameters for pure components at $25^{\circ} \mathrm{C}$ 
volume, assuming right cylinders for $n$-alkanes and spheres for other solvents. The APP chain was similarly treated as a cylinder having a crystallographic repeating length of $6.5 \times 10^{-8} \mathrm{~cm}$ for three monomer units ${ }^{12}$ and a radius of $3.15 \times 10^{-8} \mathrm{~cm}$, as required by the characteristic molar volume $V_{2}^{*}$.

\section{RESULTS AND DISCUSSION}

\section{Heats of Mixing and the $X_{12}$-Parameter}

The heats of mixing of APP at infinite dilution, $h_{\infty}^{\mathrm{E}}$, in various solvents are listed in Table II. All the heats are positive except for $n$-pentane and $n$ hexane. These results can be visualized by plotting the observed $h_{\infty}^{\mathrm{E}}$-values against the carbon atom number of each solvent, as in Figure 1, where the values for $\mathrm{CHCl}_{3}$ and $\mathrm{CCl}_{4}$ have been inserted in the empty space of the figure. For the linear alkanes, the $h_{\infty}^{\mathrm{E}}$-values are very small, as expected in view of the similarity of solute and solvent. A regular increase in heat is observed as the solvent chain length increases. The heats which are initially exothermic become endothermic for longer alkanes. Similar trends have been observed for various bulk polymers including APP in linear alkanes., ${ }^{1,5,13-15}$ However, for the aromatic solvents, the $h_{\infty}^{\mathrm{E}}$-values are very large and decrease with the introduction of

Table II. Heats of mixing at infinite dilution of atactic polypropylene at $25^{\circ} \mathrm{C}$

\begin{tabular}{|c|c|c|c|c|}
\hline \multirow{2}{*}{ Solvent } & $h_{\infty}^{\mathrm{E}}$ & $f(\alpha, \tilde{v})^{\mathbf{a}}$ & $g\left(X_{12}\right)^{\mathrm{a}}$ & $X_{12}$ \\
\hline & $\mathrm{J} \mathrm{g}^{-1}$ & $\mathrm{~J} \mathrm{~g}^{-1}$ & $\mathrm{~J} \mathrm{~g}^{-1}$ & $\mathrm{~J} \mathrm{~cm}^{-3}$ \\
\hline$n$-Pentane & -4.7 & -5.2 & 0.5 & 0.8 \\
\hline$n$-Hexane & -1.4 & -2.8 & 1.4 & 2.3 \\
\hline$n$-Heptane & 0.5 & -1.8 & 2.3 & 3.5 \\
\hline$n$-Octane & 1.0 & -1.2 & 2.2 & 3.3 \\
\hline$n$-Nonane & 2.4 & -0.8 & 3.3 & 5.0 \\
\hline$n$-Decane & 3.1 & -0.6 & 3.7 & 5.5 \\
\hline Cyclohexane & 3.9 & -1.6 & 5.4 & 7.8 \\
\hline Benzene & 30.8 & -1.6 & 32.4 & 49.9 \\
\hline Toluene & 16.5 & -0.7 & 17.2 & 24.4 \\
\hline Ethylbenzene & 14.2 & -0.4 & 14.7 & 20.0 \\
\hline$o$-Xylene & 13.4 & -0.3 & 13.7 & 18.7 \\
\hline$m$-Xylene & 12.3 & -0.3 & 12.6 & 17.3 \\
\hline$p$-Xylene & 10.3 & -0.4 & 10.7 & 13.9 \\
\hline $\mathrm{CHCl}_{3}$ & 17.2 & -1.9 & 19.1 & 28.5 \\
\hline $\mathrm{CCl}_{4}$ & 6.6 & -1.5 & 8.1 & 12.8 \\
\hline
\end{tabular}

a $f(\alpha, \tilde{v})$ and $g\left(X_{12}\right)$ show the first and second terms of the right-handed side of eq 2. $h_{\infty}{ }^{\mathrm{E}}=f(\alpha, \tilde{v})+g\left(X_{12}\right)$. aliphatic groups into benzene, suggesting that the interactions with APP are appreciably different in linear alkanes and aromatics. For cyclohexane, $\mathrm{CHCl}_{3}$ and $\mathrm{CCl}_{4}$, the heats exhibit the intermediate values of the above homologs.

According to the free volume theory, the experimental $h_{\infty}^{\mathrm{E}}$ arises from two contributions: the equation-of-state contribution, the first term on the right-hand side of eq 2, originating from the difference in free volume or thermal expansibility between polymer and solvent, and the interaction contribution including the $X_{12}$-parameter, the second term, arising from the difference in force field. The $X_{12}$-parameter can be obtained by fitting the experimental $h_{\infty}^{\mathrm{E}}$ to eq 2. Table II lists the values of $h_{\infty}^{\mathrm{E}}$, the equation-of-state term $f(\alpha, \tilde{v})$, the interaction term $g\left(X_{12}\right)$ and the $X_{12}$-parameter. The equation-of-state term is negative for all solvents. Especially for the linear alkane systems, the observed change in the $h_{\infty}^{\mathrm{E}}$-value with increasing the chain

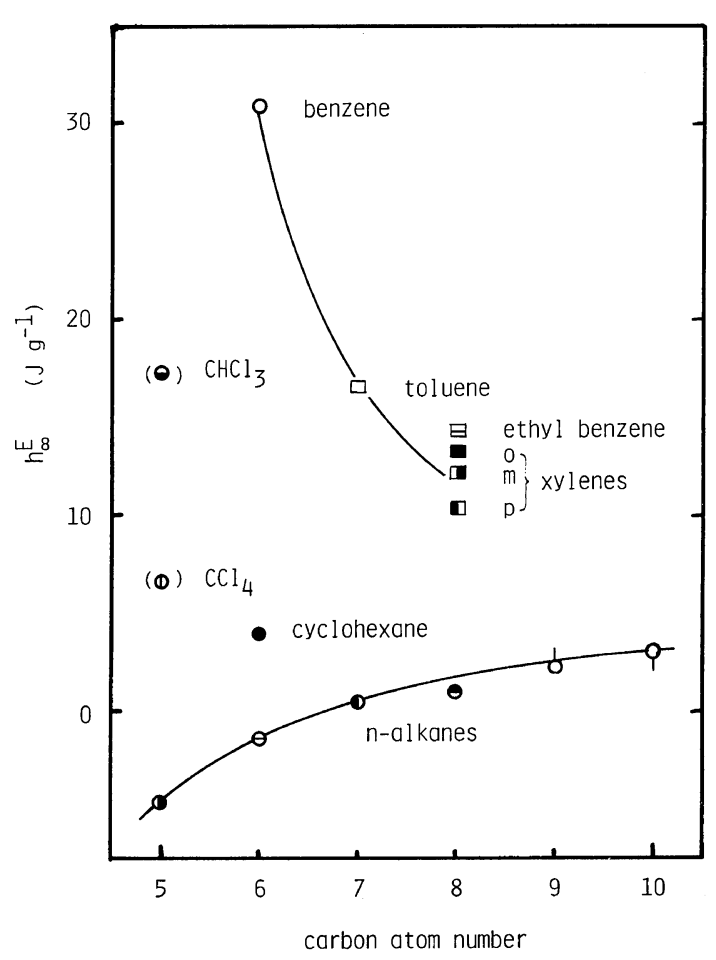

Figure 1. Heats of solution of APP at infinite dilution as a function of the carbon atom number of the solvent. The abscissa scale for $\mathrm{CHCl}_{3}$ and $\mathrm{CCl}_{4}$ is arbitrary. Symbols for solvents are the same as those appearing in Figures 1 to 5 . 
length can be explained as the gradual disappearance of the exothermic contribution toward the heat, leaving only the positive contribution for longer alkanes. But for the aromatic systems, the free volume contribution is minor and the major contribution is the interaction term. For the cyclohexane, $\mathrm{CCl}_{4}$, and benzene systems, the equationof-state terms are nearly equal to each other $\left(\sim-1.6 \mathrm{~J} \mathrm{~g}^{-1}\right)$, and therefore the difference in the heats may be ascribed mainly to the force field difference between APP and each solvent component.

As with the $h_{\infty}^{\mathrm{E}}$-value, the $X_{12}$-value increases with increasing chain length and decreases by introducing aliphatic groups into benzene. The dependence of the $X_{12}$-value on solvent properties has been examined by many authors. For alkane mixtures, although there are some difficulties, this dependence is generally ascribed to the small difference in the force field surrounding the methyl-end groups and the methylene interior groups of the components. ${ }^{16-18}$ In the present systems also; it seems relevant to discuss the dependence of $X_{12}$ in terms of the methyl-methylene and aliphatic-aromatic contacts. Thus the present $X_{12}$-values are plotted against the methyl surface fraction of solvent for the alkane series (Figure 2a), and against the aromatic surface fraction for the aromatic series (Figure $2 b$ ). The methyl and aromatic surface fractions can be evaluated from the tabulation by Bondi. ${ }^{19}$ The present scale of the abscissa seems more reasonable than the carbon atom or segment numbers of solvent molecules, since the number of contacts on mixing will be in proportion to the surface area of each component.

As can be seen from Figure 2, the plots are smooth for both the alkane and aromatic series, which incur a gradual change in force field surrounding the polymer segments. For the aromatic series, the introduction of aliphatic substituents on benzene greatly reduces the $X_{12}$-value as well as the heat of mixing. This suggests that the contact interchange of an aromatic ring with APP is very large, and that the $X_{12}$-value for more highly substituted benzene will approach a value comparable to that for alkanes. In the case of the alkane series, the $X_{12}$-value increases with a decrease the methyl surface fraction (or increasing the chain length). A smoothed extrapolation to the same methyl surface fraction as that of APP ( $\downarrow$ APP) gives a value of $X_{12}$
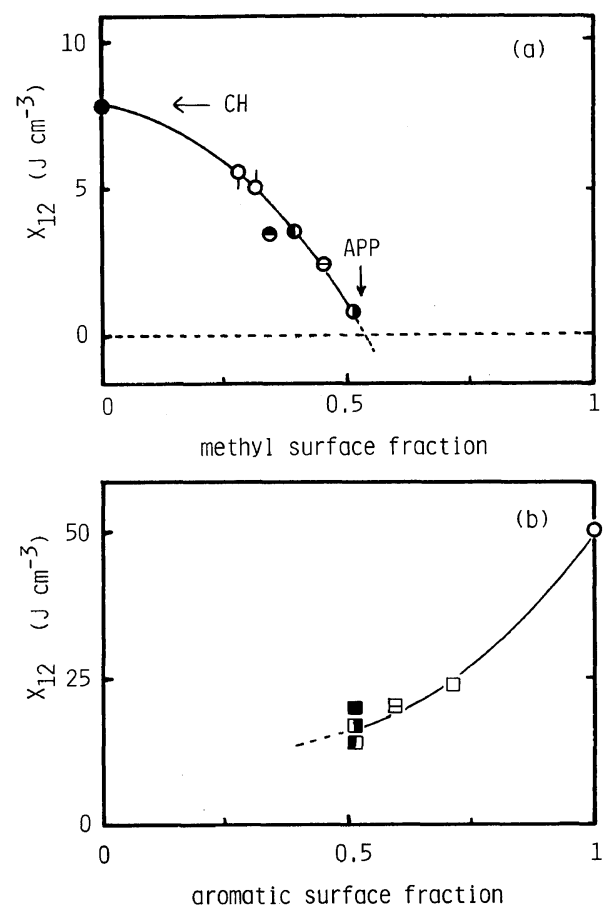

Figure 2. The dependence of $X_{12}$ on (a) the methyl surface fraction and (b) the aromatic surface fraction of solvent. $\leftarrow \mathrm{CH}$ and $\downarrow$ APP signify extrapolations to the same methyl surface fractions as cyclohexane and polypropylene, respectively.

equal nearly zero. Another extrapolation to a very long polymethylene chain gives a value of $X_{12}$ corresponding to that of cyclohexane $(\leftarrow \mathrm{CH})$ which consists of all methylene segments, although cyclohexane is not linear. From these extrapolations of $X_{12}$ for the methyl surface fraction, some interesting conclusions can be deduced: i) the $X_{12}$-value will be practically zero for mixtures in which the solvent has the same contact surface as that of the polymer, ii) the methyl contacts with APP make an appreciable negative contribution to the $X_{12}$-value, while the methylene contacts contribute positively. In fact, Delmas et $a l .{ }^{5}$ found in their calorimetric studies for the APP-alkane systems that the $X_{12}$ value is rather negative for the branched alkanes having a methyl surface fraction higher than the corresponding linear alkanes and APP itself. For PIB- $n$-alkane systems similar to the present systems, however, the dependence of $X_{12}$ on the chain length shows as opposite trend, the $X_{12}$-value is 
larger for shorter alkanes, though the $h_{\infty}^{\mathrm{E}}$-value increases with increasing chain length, as in the case of the APP systems. ${ }^{5,13,14}$ This is considered due to an overestimation of the equation-of-state term, where the calculated value of $f(\tilde{v}, \alpha)$ is appreciably negative due to a large difference in expansibility between shorter alkanes and PIB $[\alpha(\mathrm{PIB})=$ $5.55 \times 10^{-4} \mathrm{~K}^{-1}$, while $\alpha($ APP $\left.)=8.06 \times 10^{-4} \mathrm{~K}^{-1}\right] .^{5}$

\section{Excess Volume of Mixing}

Density measurements were carried out for mixtures of APP with various solvents which were three linear alkanes ( $n$-pentane, $n$-hexane, $n$-heptane) and four rather globular solvents (cyclohexane, $\mathrm{CCl}_{4}$, $\mathrm{CHCl}_{3}$, benzene). The excess volumes $\Delta v / v^{0}$ are shown in Figure 3 as functions of composition. For the linear alkanes, the excess volumes are negative and the absolute values decrease with increasing chain length. Similar volume changes have been found for the PIB-linear alkane systems. ${ }^{13}$ For rather globular solvents, the excess volumes are positive and increase in order of cyclohexane $<\mathrm{CCl}_{4}<\mathrm{CHCl}_{3}<$ benzene. The theoretical dependence of the excess volume on composition was

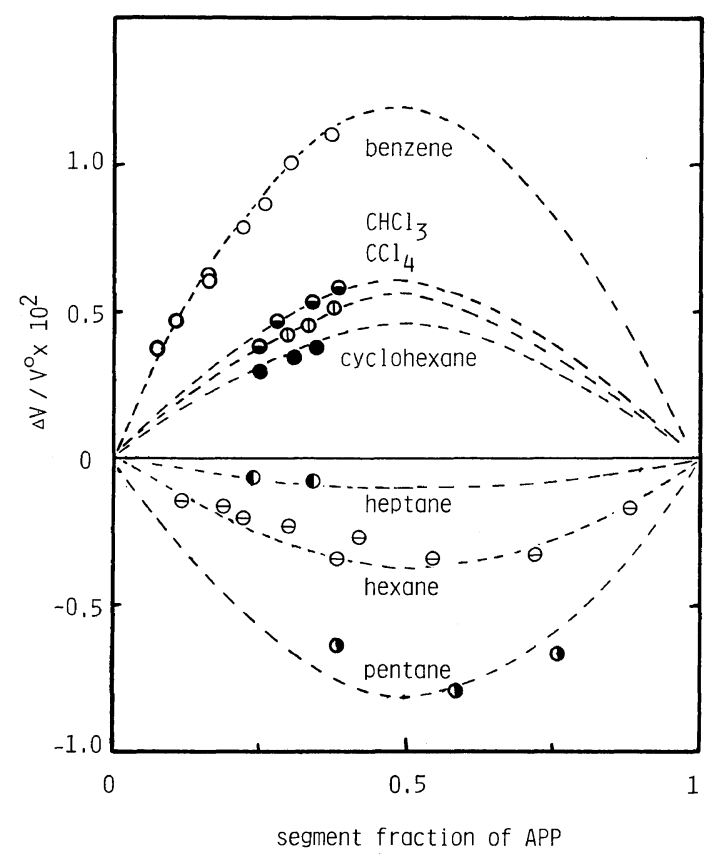

Figure 3. Excess volumes of mixing as functions of the segment fraction of APP. The dashed curves are drawn through the experimental points. calculated for each system, using eq 4-6 and the corresponding $X_{12}$-value listed in Table II. The theory correctly predicts the sign of the excess volume, which is negative for the linear alkanes and positive for the globular solvents, in agreement with observation. However, the magnitude of the theoretical value was negatively overestimated for the linear alkanes, and positively underestimated for the globular solvents (see Figure 4). Since the excess volume is not sensitive to the $X_{12}$-value, improvement in the theoretical value is very difficult, using only a small change in $X_{12}$. For example, if we tried to fit the theoretical value to that observed, a very large value of $X_{12}$ would be necessary: $17 \sim 25$ $\mathrm{J} \mathrm{cm}^{-3}$ for linear alkanes; 35 for cyclohexane; 30 for $\mathrm{CCl}_{4} ; 80$ for benzene. For various polymer solutions, it has been reported that the prediction of the excess volume by the $X_{12}$-value determined from the heat of mixing is poor. ${ }^{13,15,20-23}$

Generally, the volume change on mixing has been interpreted in terms of the difference in free volume between polymer and solvent; this difference arises mainly from the difference in the respective reduced volumes or thermal expansion coefficients. In fact, in the present APP-linear alkane systems, a good correlation was observed between the observed excess volume and the difference in the reduced volumes of APP and solvent alkane, i.e., the larger difference in the reduced volume leads to a more negative excess volume. It seems that in these systems, a closer fitting of solvent molecules to the polymer chain can be easily attained by an effective utilization of the large free volume difference. On the other hand, in rather globular solvent systems, in which the solvents have similar reduced volumes or thermal expansion coefficients, this interpretation is not suitable; the observed excess volume increases in order of cyclohexane $<\mathrm{CCl}_{4}<\mathrm{CHCl}_{3}$ $<$ benzene. This means that there is a contribution to the volume change other than that of the free volume difference. In this regard, it is of interest that both the theoretical and observed excess volumes have a rather good correlation with the $h_{\infty}^{\mathrm{E}}$ - or $X_{12}$-values for all the solvent systems studied. In Figure 4, the excess volumes interpolated to $\varphi_{2}=0.5$ are plotted against the logarithm of $X_{12}$. The plots exhibit nearly linear and parallel relations in both experiment and theory, though as mentioned before the discrepancy between them is large. We can easily see the change in the excess volume from negative to 


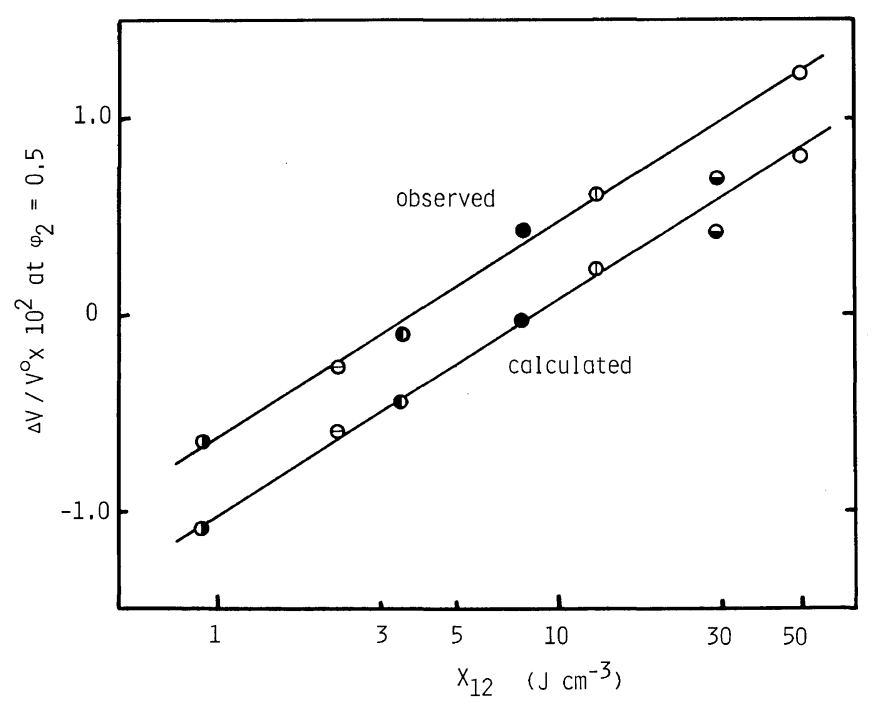

Figure 4. Relation between the excess volume interpolated at $\varphi_{2}=0.5$ and the $X_{12}$-value determined from the heat of mixing.

positive on increasing the $X_{12}$-value. This means that, although the excess volume is not very sensitive to the $X_{12}$-values, the theory does to a certain extent reflect the effect of the force field difference on the excess volume through $\tilde{T}$ including $X_{12}$ (eq 6). However, at the present, we can give no reasonable interpretation for the large discrepancy between theoretical and experimental excess volumes.

\section{The $\chi$-Parameter}

The $\chi$-parameter was determined from the vapor sorption experiments for five solvent systems ( $n$ pentane, $n$-hexane, $n$-heptane, cyclohexane, and benzene), using the familiar Flory-Huggins equation. The segment fraction was used instead of the usual volume fraction. Figure 5 shows the results for the $n$-hexane, cyclohexane, and benzene systems. The results for $n$-pentane and $n$-heptane (not shown in the figure) are similar to those for $n$-hexane except that the $\chi$-parameters are very slightly smaller for longer alkanes. The calculated $\chi$-parameters were obtained using eq 3 , the corresponding equation-ofstate parameters and the $X_{12}$-value determined from the heats of mixing $\left(Q_{12}=0\right.$ in Table III). The results are shown by the dotted lines in the figure. Agreement between calculation and experiment was not very good in any of the systems studied nor in cases of excess volume. For linear alkanes and cyclohexane, the calculated values exhibit a somewhat smaller concentration dependence of the $\chi$ -

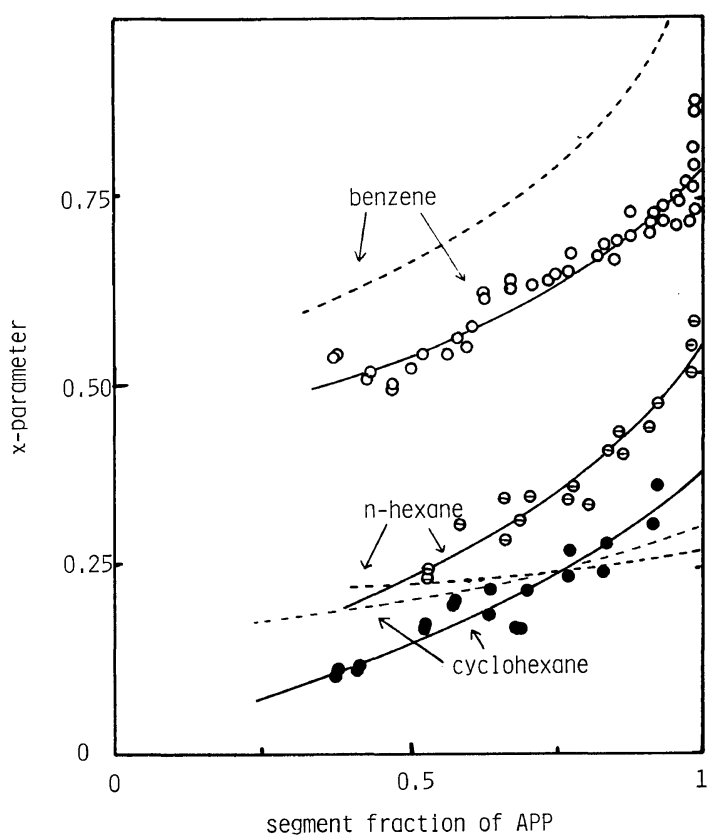

Figure 5. The $\chi$-parameter as a function of segment fraction of APP. The solid curves are described by eq 3 with the values listed in Table III (see text). The dashed curves are calculated by theory with $Q_{12}=0$. 
Thermodynamics of Polypropylene Solutions

Table III. Exchange interaction parameters

\begin{tabular}{|c|c|c|c|c|c|}
\hline \multirow{3}{*}{ Solvent } & \multicolumn{2}{|c|}{$Q_{12}=0$} & \multicolumn{3}{|c|}{ Best fit } \\
\hline & $X_{12}$ & & $X_{12}$ & $Q_{12}$ & \\
\hline & $\mathrm{J} \mathrm{cm}^{-3}$ & & $\mathrm{~J} \mathrm{~cm}^{-3}$ & $\mathrm{~J} \mathrm{~cm} \mathrm{~cm}^{-3} \mathrm{~K}^{-1}$ & \\
\hline$n$-Pentane & 0.8 & 0.59 & 1.26 & -0.021 & 0.40 \\
\hline$n$-Hexane & 2.3 & 0.61 & 3.47 & -0.021 & 0.40 \\
\hline$n$-Heptane & 3.5 & 0.63 & 5.27 & -0.025 & 0.42 \\
\hline Cyclohexane & 7.8 & 0.68 & 11.3 & -0.004 & 0.54 \\
\hline Benzene & 49.9 & 0.65 & 43.5 & +0.033 & 0.73 \\
\hline
\end{tabular}

parameter than that observed. For benzene, the theory correctly predicts the concentration dependence of the $\chi$-parameter, but overestimates the magnitude.

Following Flory ${ }^{4,13}$ we introduced a correction parameter $Q_{12}$ into the $X_{12}$ term of eq 3 as $\left(X_{12}-\tilde{v} T Q_{12}\right)$, where $X_{12}$ represents the exchange enthalpy parameter and $Q_{12}$ implies the corresponding entropy parameter. Calculations using only the $Q_{12}$ parameter did not reproduce the observed concentration dependence of the $\chi$-parameter. Thus, it was necessary to adjust the ratio $\left(s_{2} / s_{1}\right)$, since the dependence of $\chi$ on composition is very sensitive to $\left(s_{2} / s_{1}\right)$ owing to the change in the site fraction $\theta_{2}=\varphi_{2}\left(s_{2} / s_{1}\right) /\left[\varphi_{1}+\varphi_{2}\left(s_{2} / s_{1}\right)\right]$. Therefore, in order to reproduce both the experimental $\chi$ and $h_{\infty}^{\mathrm{E}}$, calculations were perform by taking $X_{12}, Q_{12}$, and $\left(s_{2} / s_{1}\right)$ as adjustable parameters, in which the product $X_{12}\left(s_{2} / s_{1}\right)$ was made to agree with that estimated from the observed $h_{\infty}^{\mathrm{E}}$. The values of $X_{12}$, $Q_{12}$, and $\left(s_{2} / s_{1}\right)$ chosen for the best fit to the experimental $\chi$ and $h_{\infty}^{\mathrm{E}}$ are given in Table III and the calculated values of $\chi$ are shown by the solid lines in Figure 4. For the linear alkanes the best fit required not only a large negative value of $Q_{12}$ but also an appreciably smaller value of $\left(s_{2} / s_{1}\right)$ than that estimated from the surface-to-volume ratio of the respective components. Here, the values of $X_{12}$ larger than those for $Q_{12}=0$ in Table III correspond to the adjustment of $\left(s_{2} / s_{1}\right)$ with somewhat smaller values. For benzene, a large positive value of $Q_{12}$ is needed, though the values of $X_{12}$ and $\left(s_{2} / s_{1}\right)$ remain unchanged. The magnitude of $Q_{12}$ for cyclohexane is very small and the experimental $\chi$ can be reproduced only by adjustment of the $\left(s_{2} / s_{1}\right)$ or $X_{12}$ values.
Thus, it may be concluded that the Flory theory requires the introduction of the exchange entropy parameter $Q_{12}$ and/or the adjustment of the contact surface ratio $\left(s_{2} / s_{1}\right)$ in order to reproduce both the experimental $\chi$ and $h_{\infty}^{\mathrm{E}}$ values. The theoretical excess volume, however, is hardly affected by such parameter alternations. A nonzero value of $Q_{12}$ is attributed mainly to refinement of the entropy contributions, such as inaccuracies in theoretical expressions for the combinatory entropy of the mixture and/or deviations from random mixing. ${ }^{4,23}$ Recently, Shiomi et al. ${ }^{24}$ proposed a modified expression for excess thermodynamic quantities by assuming non-additivity of the external degree of freedom and adopting different core volumes of the segments for polymer and solvent. Delmas et $a .^{5,25-27}$ and Patterson et al. ${ }^{18,28,29}$ also pointed out the importance of the molecular shape-specific effects, such as the orientation and steric hindrance in the polymer and solvent components. However, the Flory expressions for a simplified model have not directed any explicit consideration to such effects. The present $Q_{12}$-value may also contain contributions from these effects so far ignored. Unfortunately, it is difficult to choose reliable value of $\left(s_{2} / s_{1}\right)$, usually estimated from crystallographic data or molecular models or group surface contributions tabulated by Bondi. The present smaller values of $\left(s_{2} / s_{1}\right)$ in alkanes suggest that the effective contact surface per polymer segment is much smaller than those of the solvent alkanes.

\section{REFERENCES}

1. G. Delmas, D. Patterson, and T. Somcynski, J. Polym. Sci., 57, 79 (1962). 


\section{H. OCHIAI et al.}

2. D. Patterson, G. Delmas, and T. Somcynski, Polymer, 8, 503 (1967).

3. P. J. Flory, J. Am. Chem. Soc., 87, 1833 (1965).

4. B. E. Eichinger and P. J. Flory, Trans. Faraday Soc., 64, 2035, 2053, 2061, and 2066 (1968).

5. H. Phuong-Nguyen and G. Delmas, Macromolecules, 12, 740 and 746 (1979).

6. H. Ochiai, Y. Nishihara, Y. Yamaguchi, and I. Murakami, J. Sci. Hiroshima Univ., Ser. A, 41, 157 (1977).

7. P. J. Tait and P. J. Liversey, Polymer, 11, 359 (1970).

8. E. Passaglia and G. M. Martin, J. Res. Natl. Bur. Stand., 68A, 273 (1964).

9. R. A. Orwoll and P. J. Flory, J. Am. Chem. Soc., 89, 6814 (1967).

10. A. Abe and P. J. Flory, J. Am. Chem. Soc., 87, 1838 (1965).

11. R. R. Dreisbach, Adv. Chem. Ser., 15, (1955), 22, (1959), and 29, (1961).

12. G. Natta, J. Polym. Sci., 16, 143 (1955).

13. P. J. Flory, J. L. Elleson, and B. E. Eichinger, Macromolecules, 1, 279 (1968).

14. G. Delmas and P. Tancréde, Eur. Polym. J., 9, 199 (1973).

15. R. S. Chahal, W. P. Kao, and D. Patterson, J. Chem. Soc., Faraday Trans. 1, 69, 1834 (1973).

16. R. A. Orwoll and P. J. Flory, J. Am. Chem. Soc., 89,
6822 (1967).

17. D. Patterson and J. M. Bardin, Trans. Faraday Soc., 66, 321 (1970).

18. V. T. Lam, P. Picker, D. Patterson, and T. Tancréde, J. Chem. Soc., Faraday Trans. 2, 70, 1465 and 1479 (1974).

19. A. Bondi, J. Phys. Chem., 68, 441 (1964).

20. S. Morimoto, J. Polym. Sci., A-1, 6, 1547 (1968).

21. A. Nakajima, F. Hamada, K. Yasue, K. Fujisawa, and T. Shiomi, Makromol. Chem., 175, 197 (1974).

22. P. J. Flory and H. Shih, Macromolecules, 5, 761 (1972).

23. P. J. Flory and H. Hücker, Trans. Faraday Soc., 67, 2258, 2270, and 2275 (1971).

24. T. Shiomi, K. Fujisawa, F. Hamada, and A. Nakajima, J. Chem. Soc., Faraday Trans. 2, 76, 895 (1980).

25. S. Turrell and G. Delmas, J. Chem. Soc., Faraday Trans. 1, 70, 572 (1974).

26. G. Delmas and Ng. T. Thanh, J. Chem. Soc., Faraday Trans. 1, 71, 1172 (1975).

27. G. Charlet and G. Delmas, Polymer, 22, 1181 (1981).

28. P. Tancréde, P. Bothorel, P. de Saint-Ramain, and D. Patterson, J. Chem. Soc., Faraday Trans. 2, 73, 15 (1977).

29. P. Tancréde, P. Bothorel, and D. Patterson, J. Chem. Soc., Farady Trans. 2, 73, 29 (1977). 